\title{
Development of a UHPLC-MS/MS method for the determination of quercetin in milk and its application to a pharmacokinetic study
}

\author{
Małgorzata Gbylik-Sikorska ${ }^{1}$, Anna Gajda ${ }^{1}$, Artur Burmańczuk ${ }^{2}$, \\ Tomasz Grabowski ${ }^{3}$, Andrzej Posyniak ${ }^{1}$ \\ ${ }^{1}$ Department of Pharmacology and Toxicology, \\ National Veterinary Research Institute, 24-100 Puławy, Poland \\ ${ }^{2}$ Department of Pharmacology, Faculty of Veterinary Medicine, \\ University of Life Sciences, 20-033 Lublin, Poland \\ ${ }^{3}$ Polpharma Biologics, 80-172 Gdańsk, Poland \\ malgorzata.gbylik@piwet.pulawy.pl
}

Received: September 4, 2018 Accepted: February 27, 2019

\begin{abstract}
Introduction: Quercetin is a polyphenolic flavonoid which has been used in traditional Chinese medicine as a natural therapeutic agent with a broad spectrum of activities (antioxidant, anticancer, neuroprotective, anti-inflammatory, antiviral and antibacterial). The aim of this study was to develop and validate a rapid and simple ultra-high-performance liquid chromatography with tandem mass spectrometry (UHPLC-MS/MS) method for the determination of quercetin in milk. Material and Methods: Sample preparation was based on a liquid-liquid extraction with $0.5 \%$ formic acid in acetonitrile. The chromatographic separation was performed on a ZORBAX SB-C18 column with methanol and $0.5 \%$ formic acid as a mobile phase. Results: The procedure was successfully validated. The mean recovery of the analyte was $98 \%$, with the corresponding intra- and inter-day variation less than $10 \%$ and $15 \%$, respectively, and the repeatability and reproducibility were in the range of $3 \%-7.2 \%$ and $6.1 \%-12 \%$, respectively. The lowest level of quantification was $1.0 \mu \mathrm{g} / \mathrm{kg}$. Conclusion: The proposed method was successfully applied in evaluating the pharmacokinetics of quercetin in milk obtained from dairy cows with clinical mastitis after intramammary administration.
\end{abstract}

Keywords: quercetin, UHPLC-MS/MS, milk, pharmacokinetics.

\section{Introduction}

Quercetin belongs to the flavonol group of flavonoids, which are natural compounds occurring in photosynthesising cells in plants. Flavonoids are of great interest, because of their various pharmacological effects (antioxidant, anticancer, neuroprotective, antiinflammatory, antiviral, and antibacterial). Due to their broad spectrum of biological activities, plant extracts containing these compounds are successfully used to treat various human diseases. In the era of prevention of more bacterial resistance to antibiotics both in humans and animals, searching for new alternative treatments is a particularly vital undertaking. Reducing the use of antimicrobial drugs is one of the most important challenges in medicine, and in this endeavour Quercetin may have great importance for veterinary medicine. In the case of mastitis, which is a major endemic disease of dairy cows manifesting as inflammation of the mammary gland, antibiotic therapy reduction is very desirable. Because of its antibacterial activities, quercetin may be used in mastitis treatment as an alternative to antibiotics. However, to carry out research into the expediency of the treatment, it is necessary to have an appropriate analytical method which allows quercetin content in milk samples to be assayed. In the literature, there are some reports describing the determination of quercetin in biological samples, but most of them focus on its determination in rat plasma or plant products $(1-3,7,9,10)$. There is no published assay for the analysis of the flavonoid in milk samples. So far, quercetin antibacterial properties against different microorganisms have been tested in humans $(4,8,11)$. Its pharmacokinetics have also been 
completely evaluated in humans $(5,6)$, but for animals there are only a few papers, mostly concerning rats $(1,2,7,9)$. The pharmacokinetic profile of any flavonoid in milk from dairy cows with mastitis has not been described so far.

In this study, we developed a quantitative analytical method for the determination of quercetin in milk samples. The method was further applied for the estimation of pharmacokinetic parameters of quercetin in milk samples and could be helpful for evaluating the application of quercetin in dairy cow mastitis therapy.

\section{Material and Methods}

Reagents. All reagents used were of an analytical grade. Acetonitrile, methanol, and formic acid were obtained from J.T. Baker (Deventer, the Netherlands). Water was deionised $\left(>18 \mathrm{M} \Omega \mathrm{cm}^{-1}\right)$ by the Millipore system (Merck, Germany). Quercetin and sulphaphenazole (an internal standard - IS) were purchased from Sigma Aldrich (USA). Polyvinylidene fluoride (PVDF) syringe filters of $0.22 \mu \mathrm{m}$ size were from Restek (USA).

Chromatography and mass spectrometry. The quercetin concentration in milk was determined using a Nexera X2 ultra-high-performance liquid chromatography-tandem mass spectrometry (UHPLCMS/MS) (Shimadzu, Japan) system connected to a QTRAP 4500 triple quadrupole mass spectrometer (AB Sciex, USA). Analyst 1.6.3 software (AB Sciex) controlled the UHPLC-MS/MS system and processed the data. The chromatography separation was performed on a ZORBAX SB-C18 column (50 $\mathrm{mm} \times$ $2.1 \mathrm{~mm} \times 1.8 \mu \mathrm{m})$ (Agilent, USA) integrated with a guard column of the same type. The mobile phase for analysis consisted of methanol (A) and $0.5 \%$ formic acid (B). Gradient elution was performed as follows: 0.0-2.0 $\mathrm{min}(15 \% \mathrm{~A}), 2.01-3.3 \mathrm{~min}(80 \% \mathrm{~A})$, and $3.31-5.0 \mathrm{~min}(15 \% \mathrm{~A})$ at a flow rate of $0.5 \mathrm{ml} / \mathrm{min}$ and injection volume of $5 \mu$ l. Detection was performed in the negative ionisation mode in the selected reaction monitoring mode (SRM). The transitions $\mathrm{m} / z 301>151$ and $301>178$ for quercetin and $313>156$ for the IS were monitored.

Preparation of standards and quality control samples. A stock solution of quercetin $(1 \mathrm{mg} / \mathrm{ml})$ was prepared in acetonitrile and stored in an amber volumetric flask at $-18^{\circ} \mathrm{C}$ for no longer than two months, and a working standard solution $(1 \mu \mathrm{g} / \mathrm{mL})$ was prepared in water and stored in a plastic flask at $4{ }^{\circ} \mathrm{C}$ for no longer than one week. A stock IS solution $(1 \mathrm{mg} / \mathrm{mL})$ was prepared in water and stored in an amber volumetric flask at $-18^{\circ} \mathrm{C}$ for no longer than six months, and a working solution $(1 \mu \mathrm{g} / \mathrm{mL})$ was prepared in deionised water and stored in an amber volumetric flask at $4^{\circ} \mathrm{C}$ for no longer than one month. Quality control (QC) samples were prepared by spiking blank milk samples at three concentration levels (10,
100 , and $1,000 \mu \mathrm{g} / \mathrm{kg}$ ) for the validation process and at six concentration levels $(1,10,100,1,000,5,000$ and $10,000 \mu \mathrm{g} / \mathrm{kg}$ ) during routine real sample analysis.

Milk samples preparation. Milk (500 mg) was weighed in a $1.5 \mathrm{~mL}$ microcentrifuge tube, then $50 \mu \mathrm{L}$ of IS and $1 \mathrm{~mL}$ of $0.5 \%$ formic acid in acetonitrile were added, mixed, and centrifuged at 9,447 $\times$ g for $5 \mathrm{~min}$ at room temperature. Supernatant was filtered through a $0.22 \mu \mathrm{m}$ PVDF syringe filter into chromatographic vials.

Validation method. The linearity, selectivity, repeatability, reproducibility, recovery, lower limit of quantification (LLOQ), and matrix effect (ME) of the method were evaluated. The linearity was determined by the matrix-matched calibration curve which was prepared by fortifying blank milk samples at 10 concentration levels $(1-10,000 \mu \mathrm{g} / \mathrm{kg})$. IS in a $50 \mu \mathrm{L}$ volume was added to each sample. Quantitative results of evaluation were obtained by comparing the analyte/IS peak area ratio from a matrix-matched calibration curve to the analyte/IS peak area ratio in analysed samples. Selectivity was checked by analysis of 20 blank milk samples collected from different sources which allows the possible presence of interfering substances to be verified around the retention times of the compounds of interest.

The repeatability was calculated as the relative standard deviation (RSDr, \%) of results obtained after fortifying six blank milk samples at three concentration levels $(10,100$, and $1,000 \mu \mathrm{g} / \mathrm{kg})$. The spiked samples were prepared and analysed on the same day with the same instrument and the same operators. The withinlaboratory reproducibility was calculated as the RSDr of the results obtained after fortifying another two sets of blank samples at the same concentration levels of analysed compounds as for the repeatability and analysing them on two different days with the same instrument and different operators.

The average recovery was evaluated in the same experiment as repeatability by comparing the mean measured concentration with the fortified concentration of the samples in relation to matrix-matched calibration curve. Validation also needed the LLOQ, and it was defined as the lowest concentration on the calibration curve determined at a signal-to-noise $(\mathrm{S} / \mathrm{N})$ of 10 .

Completing validation, the $\mathrm{ME}$ was evaluated as the ratio of analyte peak area added into postextracted blank milk samples to the IS peak area in water at corresponding concentrations $(10,100$, and $1,000 \mu \mathrm{g} / \mathrm{kg})$.

Application. The animal study was conducted with the consent of the Local Ethical Committee for Animal Experiments in Lublin (33/2017). Based on a somatic cell count $(\mathrm{SCC}>300,000$ cells $/ \mathrm{ml})$ and clinical investigation, nine dairy cows with clinical mastitis in one quarter were selected. The animals were of Polish Black-and-White breed, weighed $700 \mathrm{~kg}$ each, and were 4-12 years of age. Analysis of quercetin pharmacokinetics was performed after a single 
intramammary dose of $10 \mathrm{mg} /$ quarter. Milk samples $(5 \mathrm{~mL})$ were collected into plastic tubes at 5 and 15 min, and 4, 6, 8, 12, 24, 36, 48, 72, 96, 120, 144, 168, and $192 \mathrm{~h}$ after quercetin administration. The samples were frozen at $-20^{\circ} \mathrm{C}$ until analysis.

The pharmacokinetic investigation of quercetin was performed using Phoenix WinNonlin 7.0 (Certara, USA) and ThothPro 4.1 (ThothPro, Poland) software. SHAM analysis was based on the slope, height, and area. The area under the curve calculated between zero and the last sampling point $\left(\mathrm{AUC}_{0-\mathrm{t}}\right)$, the area under the first moment curve calculated between zero and the last sampling point $\left(\mathrm{AUMC}_{0-\mathrm{t}}\right)$, the mean residence time calculated for the last sampling point $\left(\mathrm{MRT}_{0-\mathrm{t}}\right)$, the elimination halflife $\left(\mathrm{t}_{1 / 2 \mathrm{kel}}\right)$, the elimination rate constant $\left(\mathrm{k}_{\mathrm{el}}\right)$, the maximal concentration $\left(\mathrm{C}_{\max }\right)$, the elimination half-life based on $\mathrm{MRT}_{0-\mathrm{t}}\left(\mathrm{t}_{1 / 2 \mathrm{MRT}}\right)$ and calculated using the equation $t_{1 / 2 M R T}=\operatorname{Ln}(2) /\left(1 / M_{R T} T_{0-t}\right)$, the volume of distribution in steady state $\left(\mathrm{V}_{\mathrm{ss}}\right)$, and the clearance $(\mathrm{CL})$ were determined.

\section{Results}

The chromatograms of the blank milk samples and those fortified at $10 \mu \mathrm{g} / \mathrm{kg}$ concentration are shown in Fig. 1.

The matrix-matched calibration curves showed good linearity $\left(\mathrm{r}^{2}>0.997\right)$ over the range of 10 $10,000 \mu \mathrm{g} / \mathrm{kg}$ for quercetin. The selectivity of the method was found to be satisfactory with no interfering peaks from endogenous compounds in the retention time of the target analyte in milk samples. The method was repeatable since the RSDr was in the range of 3\%$7.2 \%$, and RSDwR ranged from $6.1 \%$ to $12 \%$ for all investigated concentration levels for quercetin in milk samples.

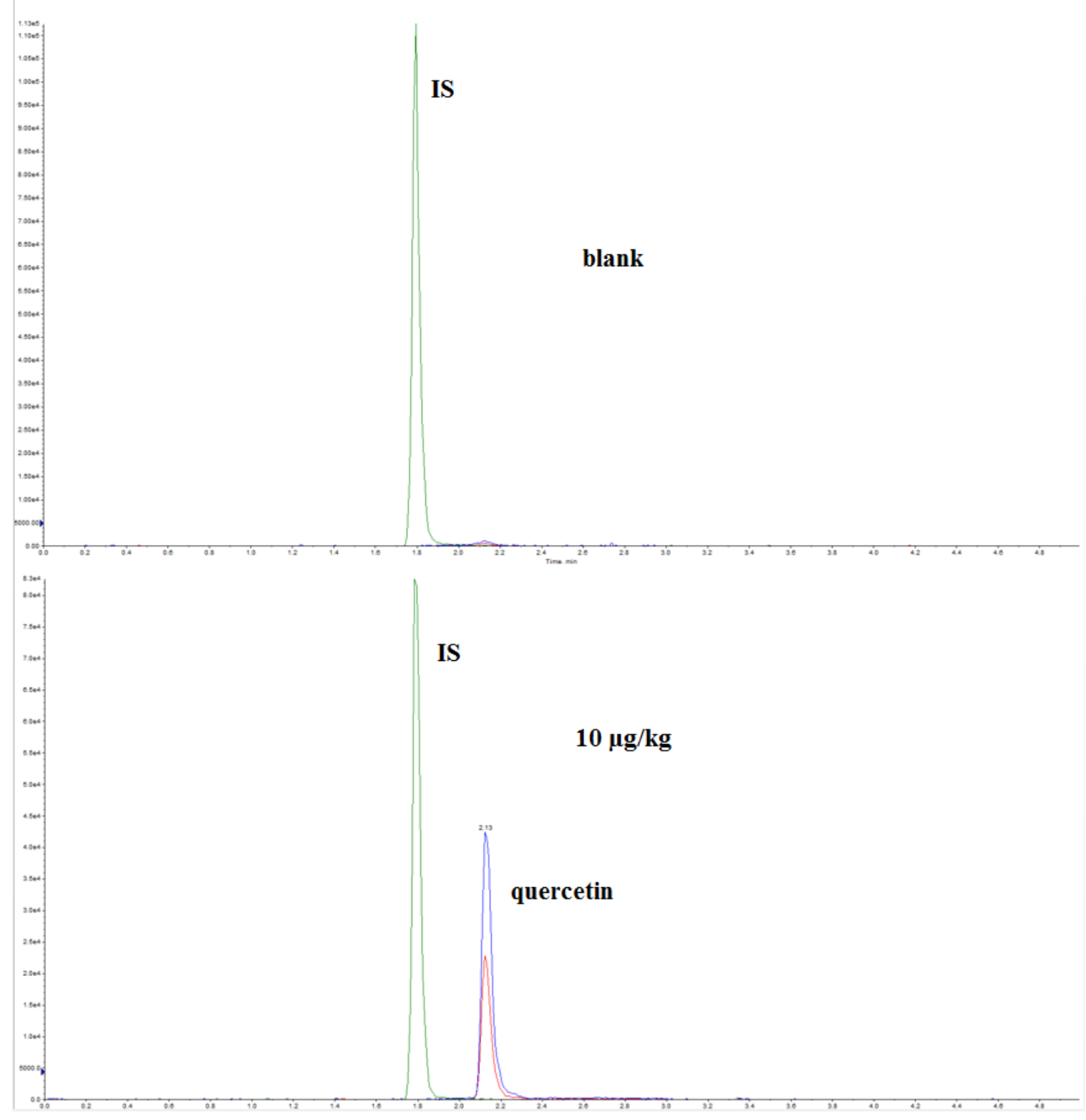

Fig. 1. Chromatograms of blank and fortified milk samples 


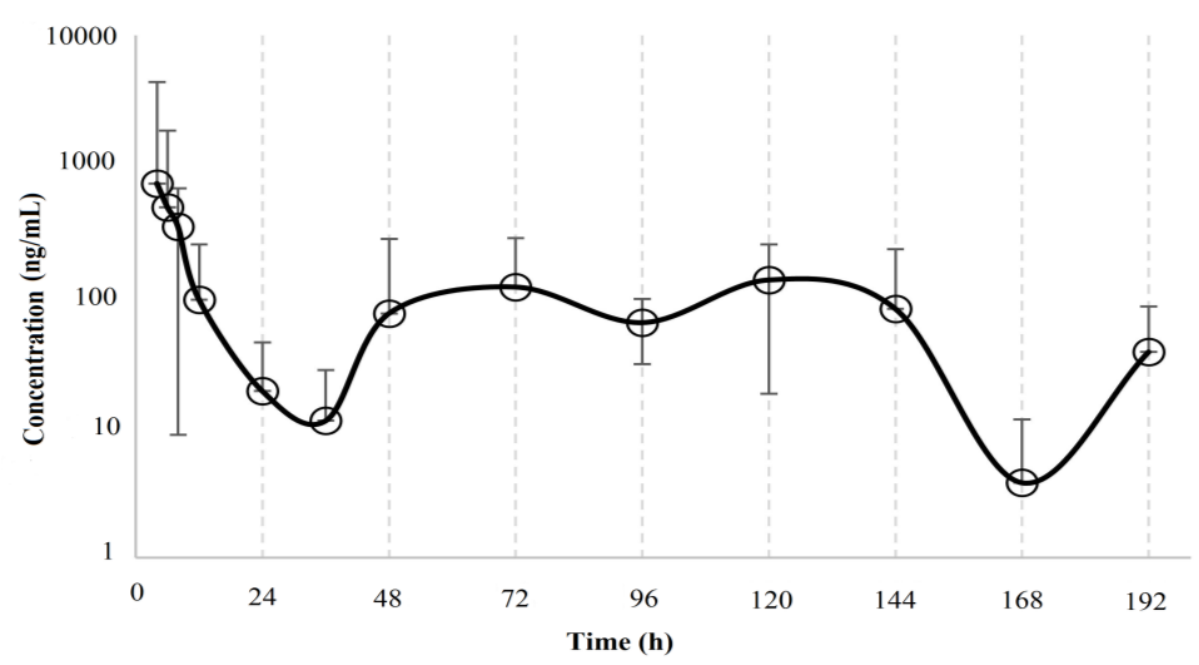

Fig. 2. Quercetin log depletion phase in milk

Satisfactory average recoveries were obtained and ranged from $89.1 \%$ to $108 \%$, depending on the concentration levels. The LLOQ of the method for quercetin was calculated as $1.0 \mu \mathrm{g} / \mathrm{kg}$. The ME was $2.6 \%, 1.8 \%$, or $2.8 \%$, depending on the quercetin concentration level. The results indicate that ME does not affect the determination of quercetin in milk samples.

The developed method was successfully applied for the determination of quercetin in milk samples. The concentration-time profile is shown in Fig. 2.

The calculated values of selected pharmacokinetic parameters were as follow: $\mathrm{AUC}_{0-\mathrm{t}} 31.64 \pm$ $17.04 \mu \mathrm{g} \times \mathrm{h} / \mathrm{g}, \mathrm{AUMC}_{0-\mathrm{t}}-1,904.47 \pm 903.10 \mu \mathrm{g} \times$ $\mathrm{h}^{2} / \mathrm{g}, \mathrm{MRT}_{0-\mathrm{t}} 67.4 \pm 25.77 \mathrm{~h}, \mathrm{t}_{1 / 2 \mathrm{MRT}} 46.72 \pm 17.86, \mathrm{t}_{1 / 2 \mathrm{kel}}$ $3.89 \pm 2.66 \mathrm{~h}, \mathrm{k}_{\mathrm{el}} 0.245 \pm 0.1351 / \mathrm{h}, \mathrm{C}_{\max } 2.80 \pm$ $3.54 \mu \mathrm{g} / \mathrm{g}, \mathrm{V}_{\mathrm{ss}} 29.94 \pm 21.15 \mathrm{l}$, and CL $0.402 \pm$ $0.207 \mathrm{~h}^{-1}$. Every $\approx 65 \mathrm{~h}$ after single administration, a fluctuation of quercetin concentrations was observed. The equable decrease in quercetin concentrations in the late elimination phase could indicate a twocompartmental model of its distribution in udder tissue. However, the decrease and increase in the concentration exclude the compartmental theory.

\section{Discussion}

The UHPLC conditions were investigated to elicit how to best separate interfering compounds in a short runtime, and the mass spectrometer parameters were optimised to receive better resolution. The following values were chosen: 90 and $100 \mathrm{~V}$ declustering potential, 27 and $33 \mathrm{~V}$ collision energy, and 13 and $15 \mathrm{~V}$ cell exit potential for quercetin and sulphaphenazole, respectively. These settings resulted in a high intensity peak for both compounds and good linearity in the regression of the calibration curves. Several mobile phases including a combination of acetonitrile, methanol, and water with or without acid were tested. Using the mixture of methanol and $0.5 \%$ formic acid resulted in the most effective separation and the best peak shapes of analytes. Various conditions of liquid-liquid extraction (LLE) were tested for isolation of quercetin from milk samples to achieve the most effective extraction procedure: acetonitrile, acetonitrile with acids (formic acid, acetic acid, and citric acid), and methanol with acetonitrile. The outcome was finding an extraction mixture of $0.5 \%$ formic acid in acetonitrile followed by $0.22 \mu \mathrm{m}$ syringe filtration suitable to allow effective protein denaturation and elimination of undesirable compounds from the extract.

The present study is the first to describe the pharmacokinetics of quercetin in milk samples collected from dairy cows with mastitis. The mean concentration of quercetin in milk samples decreases rapidly after administration. Most of the evaluated parameters show high individual variability, where for example for $\mathrm{C}_{\max }$ it is over $120 \%$, and for $\mathrm{AUC}_{0-\mathrm{t}}$ it is over $50 \%$. Because of the fast decline of quercetin concentration in milk, the $t_{1 / 2 k e l}$ is very short. It is not possible to precisely define this parameter because of fluctuations of the concentration in the late elimination phase. Therefore, the $\mathrm{MRT}_{0-\mathrm{t}}$ parameter, which in this study is much longer than $t_{1 / 2 k e l}$, can be a substitute for the presentation of the elimination phase.

In conclusion, a sensitive, selective, and rapid method for quantification of quercetin in milk samples was developed. The method was validated and applied in the determination of quercetin concentration in milk after experimental administration. Such an analytical method, suitable for the determination of quercetin levels for calculations of its pharmacokinetics in milk samples, is reported for the first time.

Conflict of Interests Statement: The authors declare that there is no conflict of interests regarding the publication of this article. 
Financial Disclosure Statement: This work was funded by the KNOW (Leading National Research Centre) Scientific Consortium in Poland "Healthy Animal - Safe Food", decision of the Ministry of Science and Higher Education (No. 05-1/KNOW2/2015).

Animal Rights Statement: The experiments on animals were conducted with the approval of the Local Ethical Committee in Lublin.

\section{References}

1. de Boer V.C., Dihal A.A., van der Woude H., Arts I.C., Wolffram S., Alink G.M., Rietjens I.M., Keijer J., Hollman P.C.: Tissue distribution of quercetin in rats and pigs. J Nutrit 2005, $135,1718-1725$

2. Chang L., Ren Y., Cao L., Sun Y., Sun Q., Sheng N., Yuan L., Zhi X., Zhang L.: Simultaneous determination and pharmacokinetic study of six flavonoids from Fructus Sophorae extract in rat plasma by LC-MS/MS. J Chrom B 2012, 904, $59-64$.

3. Coppin J.P., Xua Y., Chena H., Pan M.H., Hoc Ch.T., Juliani R., Simon J.E., Wu Q.: Determination of flavonoids by LC/MS and anti-inflammatory activity in Moringa oleifera. J. Funct. Foods 2013, 5, 1892-1899.
4. Cushnie T.P.T., Lamb A.J.: Antimicrobial activity of flavonoids. Int J Antimicrob Agents 2005, 26, 343-356.

5. Erlund I., Kosonen T., Alfthan G., Mäenpää J., Perttunen K., Kenraali J., Parantainen J., Aro A.: Pharmacokinetics of quercetin from quercetin aglycone and rutin in healthy volunteers. European J Clin Pharm 2000, 56, 545-553.

6. Graefe E.U., Wittig J., Mueller S., Riethling A.K., Uehleke B., Drewelow B., Pforte H., Jacobasch G., Derendorf H., Veit M.: Pharmacokinetics and bioavailability of quercetin glycosides in humans. J Clin Pharm 2001, 41, 492-499.

7. He J., Feng Y., Ouyang H.Z., Yu B., Chang Y.X., Pan G.X., Dong G.Y., Wang T., Gao X.M.: A sensitive LC-MS/MS method for simultaneous determination of six flavonoids in rat plasma: application to a pharmacokinetic study of total flavonoids from mulberry leaves. J Pharm Biomed Anal 2013, 84, 189-195

8. Jaisinghani R.N.: Antibacterial properties of quercetin. Microbiol Res 2017, 8, 6877 .

9. Kumar D., Verma P.R.P., Singh. S.K., Viswanathan S.: LC-ESIMS/MS analysis of quercetin in rat plasma after oral administration of biodegradable nanoparticles Biomed Chromatogr 2015, 29, 1731-1736.

10. Raote A., Jangid A., Tale R.: Liquid chromatography - tandem mass spectrometric method for simultaneous determination of rutin and quercetin from leaves of artocarpus lakoocha roxb. Int J Pharm Biol Sci 2011, 2, 848-853

11. Woźnicka E., Kuźniar A., Nowak D., Nykiel E., Kopacz M., Gruszecka J., Golec K.: Comparative study of the antibacterial activity of some flavonoids and sulfonic derivatives. Pol Pharm Society 2013, 70, 567-571. 